\title{
Using Web 2.0 Tools for Adaptive and Collaborative E-Learning
}

\author{
Katerina Papanikolaou, Stephanos Mavromoustakos \\ Dept. of Computer Science and Engineering \\ European University Cyprus \\ Nicosia, Cyprus
}

\begin{abstract}
In this paper we present an innovative framework for developing adaptive e-Learning systems based on Web 2.0 as a means to enhance the effectiveness of on-line learning systems in achieving their pedagogical goals. The proposed framework uses the Web 2.0 tool selection process in order to identify the appropriate tools for the proposed pedagogical objectives and thus improve the effectiveness of $e$ Learning systems.
\end{abstract}

\section{Introduction}

The achievement of pedagogical objectives in online learning platforms is proving increasingly difficult. Poor student motivation, lack of interest, student isolation and unfamiliarity with the use of technology are a few of the factors contributing to this.

In principle, online learning systems should be able to achieve a higher degree of student motivation and student achievement 0 . Most online learning systems though are lacking in providing the learner with these abilities, usually due to poor design and implementation as well as missing requirements 0 . Online learning platforms are usually used for supporting in-class learning and appear as material dissemination platforms. All learners are presented with the same interface and the same tools. Although communication is enabled, it is usually not adaptive to user needs and competences. Assessment tools are also presented without differentiation to all users. Finally, the systems are not able to support selfpaced learning adaptive to user learning rate and lifestyle. In a diverse learner group the online learning platform should serve as an adaptive learning tool rather than a supportive tool.

Online learning software has to adhere to the software quality factors and standards. The advent of Web 2.0 tools and technologies is enhancing the interactivity of online learning platforms, but care should be taking not to deviate from the pedagogical objectives 0 . Most learners are already familiar with Web 2.0 tools and technologwies and will be able to use them for enhancing their learning experience. Web 2.0 tools can be used for informal information and experience sharing 0 . Instructors may use such tools to attract learner attention and improve motivation. Additionally, multimedia tools can be used to attract learner interest in the form of videos and pre-recorded material used for recording lectures and tutorials, thus conveying some of the lecture qualities to the virtual environment 0,0 .

This paper is organized as follows: section II presents the Web 2.0 enhanced pedagogy requirements, while section III explains the Web 2.0 tools and their use in e-Learning. Section IV demonstrates the e-Learning Development process with the inclusion of Web 2.0, and finally section $V$ draws the concluding remarks and suggests future work.

\section{Web 2.0 enhanced pedagogy requirements}

The online learning platform should be able to provide the learner with individually defined and adaptive learning paths. It is often the case that online learners come from different and diverse backgrounds with varying competences and skills. A uniformity of background competences and skills may be achieved through the use of especially designed material, where each learner selects and follows a different learning path. The learner should also be able to create a personalized schedule according to the time available for studying. Personalized schedules will allow learners to make better use of their time and thus retain interest and increase motivation.

In order to achieve the successful transferring of the real world classroom to the new Web 2.0 enhanced learning environment we have to assess and evaluate the advantages offered by each proposed Web 2.0 learning tool 0 . The aim of using the new tools is to keep students motivated in their learning activities thus helping student retention and the achievement of the courses' pedagogical objectives. Students should maintain the feeling of individuality and differentiation; adapt their learning pace to their own individual schedule and ability whilst being part of the online learning community. The key factors that need to be addressed in the Web 2.0 pedagogical environment are identified as follows:

- The identification of learners' needs - In the e-Learning environment each student may identify his own needs.

- The structuring of the pedagogical material - The construction of the 
pedagogical material should be done so that it can expand and contract according to the learner's needs

- The enhancement of e-Learning with Web 2.0 tools - In the purely virtual environment the social activities cultivated in the realworld classroom are transferred using the Web 2.0 tools. Both instructors and students can use the tools and applications that best fit their goals. The use of these tools will contribute to the creation of an environment highly conductive to learning.

- $\quad$ The motivation for student participation The transferring to the virtual environment is not always straight forward and easy. Students are not always willing to use the virtual environment for a number of reasons, such as the difficulty of the e-Learning platform, the non-intuitive nature of the environment, the provision of reduced interactivity, etc. The addition of the Web 2.0 tools should provide the mechanisms for similar or even new social activities among students and instructors. The inclusion of Web 2.0 tools will help the dissemination of explicit or tacit information among the learners

- The establishment of collaborative mechanisms among students - The virtual environment is prone to creating isolated learners hiding behind a screen. This feeling of isolation greatly demotivates the students. This is easily avoided in the real classroom and should be avoided in the virtual classroom too, by organizing and operating in a collaborative basis so that students can interact and communicate. The use of Web 2.0 tools is of paramount importance in this factor.

- The utilization of the relevant tools and components for the support of any specific solution - Depending on the targeted student audience and the required learning outcome the appropriate tools should be implemented and differentiated accordingly. Vocational training requires different solutions than educational training and undergraduate training has different pedagogical targets than postgraduate training. Tools and components can be utilized to enhance the ELearning environment more efficiently 0.

- The right mix of the learning processes implemented - The most important learning processes are identified as follows: analysis, synthesis, reasoning, judging, problem solving, collaboration, simulation, evaluation, presentation and relation. These processes should be used dynamically for constructing the learning scene for each course and student.

\section{Web 2.0 tools for e-Learning}

The inclusion of the Web 2.0 tools should not be performed arbitrarily 0 . The designer and the educator should be able to select the Web 2.0 tools most fitting to the specified pedagogical requirements and objectives. These will be placed in three categories: information dissemination, selfpaced learning and evaluation 0 .

The inclusion of Web 2.0 tools aims at keeping learners highly motivated and connected to the learning community 0 . The learner will feel confident and retain interest in advancing his/her knowledge. Fast paced systems create learners who cannot keep up with the rest of the class, lose interest, perform badly and quit 0 . Slow paced systems create bored learners who lose motivation and interest. A well-paced evaluation system with frequent rewards and clearly defined goals creates motivated and dedicated learners; such a system will also regulate the learner's progress according to achievement. The feeling of isolation and "being left behind" is easily created in the virtual environment, especially for learners who are not used in the use of the new technologies. The right mix of technologies and methods will help overcome this. The appropriate Web 2.0 tools will allow learners to maintain motivation and keep up with the pace of the rest of the class. The Web 2.0 tools cover the need for communication and socialization in the Internet. None of the Web 2.0 tools as described in the next section were developed for educational or training purposes, but they can be used as part of the eLearning process in order to improve its effectiveness in achieving the pedagogical objectives set by the trainers. In Table 1 we present the basic tools and their characteristics. The developer / trainer should be able to assess and evaluate the tools that will best serve the preset goals taking into consideration the intended audience, the level of the training, the environment the training is intended for, etc.

\section{Web 2.0 tools}

Social networking applications and services on the Web have changed the way we perceive, use and interact on the Internet 0 . Software engineers have used a number of these services for a long time, but their value multiplies as part of the Web 2.0 ecosystem 0,0,0. Web 2.0 tools can be classified according to purpose, use and popularity. The major categories that are identified are as follows: discussion, collaboration, publishing, relationbuilding, subscription and code exchange. The tools we present next are representative of these 
categories. Here we have to take into account the effect of combining two or more of these tools.

TABLE I. WEB 2.0 SERVICES AND THEIR APPLICATIONS

\begin{tabular}{|l|l|l|l|}
\hline \multicolumn{4}{|c|}{ Web 2.0 services and their applications } \\
\hline Service & \multicolumn{1}{|c|}{ Purpose } & \multicolumn{1}{c|}{ Use } & \multicolumn{1}{c|}{ Applications } \\
\hline Forums & Discussion & $\begin{array}{l}\text { Very } \\
\text { high }\end{array}$ & $\begin{array}{l}\text { sourceforge.net } \\
\text { www.phpbb.com }\end{array}$ \\
\hline Wiki & Collaboration & High & $\begin{array}{l}\text { www.mediawiki.org } \\
\text { www.wikia.org }\end{array}$ \\
\hline Blogs & Publishing & $\begin{array}{l}\text { Very } \\
\text { high }\end{array}$ & $\begin{array}{l}\text { wordpress.com } \\
\text { www.blogger.com }\end{array}$ \\
\hline $\begin{array}{l}\text { Podcasts } \\
\text { and video } \\
\text { podcasts }\end{array}$ & Publishing & $\begin{array}{l}\text { Very } \\
\text { high }\end{array}$ & $\begin{array}{l}\text { www.apple.com/itunes } \\
\text { www.youtube.com }\end{array}$ \\
\hline $\begin{array}{l}\text { Social } \\
\text { networks }\end{array}$ & Relation & $\begin{array}{l}\text { Very } \\
\text { high }\end{array}$ & $\begin{array}{l}\text { www.myspace.com } \\
\text { www.facebook.com } \\
\text { www.linkedin.com }\end{array}$ \\
\hline $\begin{array}{l}\text { RSS } \\
\text { Public } \\
\text { code } \\
\text { repositori } \\
\text { es }\end{array}$ & Code & Medium & $\begin{array}{l}\text { www.google.com/reader } \\
\text { www.bloglines.com }\end{array}$ \\
\hline
\end{tabular}

\section{Forums}

Forums were one of the first tools for expressing collective opinion even before Web 2.0 was created. The Web 2.0 forums have gained a new role. Modern forums provide a meeting point for collaboration. Forums are not used for referring to another website or to an internal webpage; instead they act as a form of starting point of a discussion or communal task. Forums are the easiest way to start working actively in Web 2.0 since the expression of opinion can start by clicking on the reply button. Edit tools with graphical user interfaces allow ease of use whilst providing functional representation. Forums main use is for discussion and the frequency of use is very high.

\section{Wikis}

The invention of wikis came to answer the question of how to share and capitalize on the use of shared knowledge. Wikis are not forums or content management tools. Wikis now form the main and best Web 2.0 collaboration tool for creating and editing content online. Wikipedia is the most famous example of a Web 2.0 application. The effect of wikis is so profound that the term wikinomics was coined to describe a new economy based on the ability to create value in a collaborative, distributed process.

The wiki uses its own syntax. Some people complain about learning a Web language different from HTML, but the wiki syntax is a current standard, and it's simpler than HTML for nonprogrammers to learn. Wiki users can begin editing almost immediately. Newer applications are using GUI interfaces that overcome this problem. Users can use the interfaces without any previous knowledge or training. Wiki applications also let users see each document's history, compare its different versions, and undo specific changes. Users are able to create and share their own notes and resources and actively participate in a knowledge management environment where they can transfer knowledge and experiences. The main purpose for using for wikis is collaboration and their use is high.

\section{Blogs}

Blogs were the key applications for the socialization of the Internet that we typify by the term Web 2.0. The number of registered blogs is more than 100 million, and starting a new one takes only a few seconds. A student can use the blogosphere to broadcast her messages to other students without intermediaries. Blogs, wikis, and forums form the main Web 2.0 collaboration tools for projects, except in the coding phase. Podcasts and video podcasts have swamped the Web with media content and now drive the evolution of blogs. Blogs are mainly used for publishing and their use is very high.

\section{Social Networks}

The creation of online social networks multiplied the degree of directness the Internet can offer. Users of social networks now feel connected to their peers. The time spent by users in the social networks is a testimony to this 0. Applications such as MySpace, Facebook, and Twitter at first addressed mainly private users, but they're now used popular as professional networks, following the success of more business-oriented networks such as LinkedIn. Social networks are now used for marketing purposes. They have brought positive and negative influences especially due to their excessive and sometimes abusive use. The debate continues and there is a clear need for establishing the correct guidelines for their use. The main use of social networks is to create relations and groups among participant. Social networks use is extending to the disseminating of information among groups centered on common interests 0 . Their use is ranked high and their stickiness even higher due to additional services offered.

\section{RSS}

Really Simple Syndication (RSS) helps users manage and order the superabundance of information, in the Web. Customers, employees, and partners can subscribe to RSS feeds to receive current company information, news, offers, etc. automatically. RSS plays an important role in keeping companies up to date on what is happening in the market with customers and competitors. Their main use is for subscribing and their use is medium. 


\section{Public Code Repositories}

Code repositories are not a new concept to Web 2.0. They are inherent from software engineering, but they have acquired a new aspect in the open collaboration of Web 2.0 development. The code is no longer an asset protected by secrecy; it is now perceived as the element that lets others collaborate with you.

Public code repositories open the entire software development process, giving participants feedback almost immediately. A company can start by publishing its roadmaps, letting users add ideas and vote for their preferences. Likewise, a company can publish project requirements and engage users and external collaborators in refining them. This gives coding-process tools functionalities similar to those of wikis. Current integrated development environment (IDE) tools can connect with the public code repositories, making the source code available to other developers in other IDEs. Their main use is to share code and their use is high.

\section{Web 2.0 tool inclusion in the e- Learning platform development process}

The e-Learning engineering process includes the Web 2.0-enhanced e-Learning Pedagogy Requirements and the Web 2.0 tools are described in the previous sections. This process is based on the SpiderWeb software development process 0,0 that is modified to include the additional requirements of designing and developing e-Learning systems with Web 2.0 functionality. The process includes six phases (Fig. 1): Formulation, Planning, Analysis, Engineering, Implementation \& Testing, and Faculty/Student Evaluation.

\section{Formulation}

In the Formulation phase, the tasks and goals of the e-Learning application are specified and defined. The length of the first increment is also specified in the same phase. In this phase, the required Web 2.0 functionality needs to be defined. The e-Learning application may be used as an autonomous system offering virtual courses, or in parallel with the existing real classroom courses. The Web 2.0 tools that will support the e-Learning environment will be selected here according their attributes and specified pedagogical goals. The clear identification of these goals is crucial for identifying the real system needs and requirements resulting to the development of an e-Learning system. A single tool or a combination of tools may be selected such as content-management platforms combined with Weblogs. In the same sense, the pedagogy material should be developed and produced based on the formulated application goals.

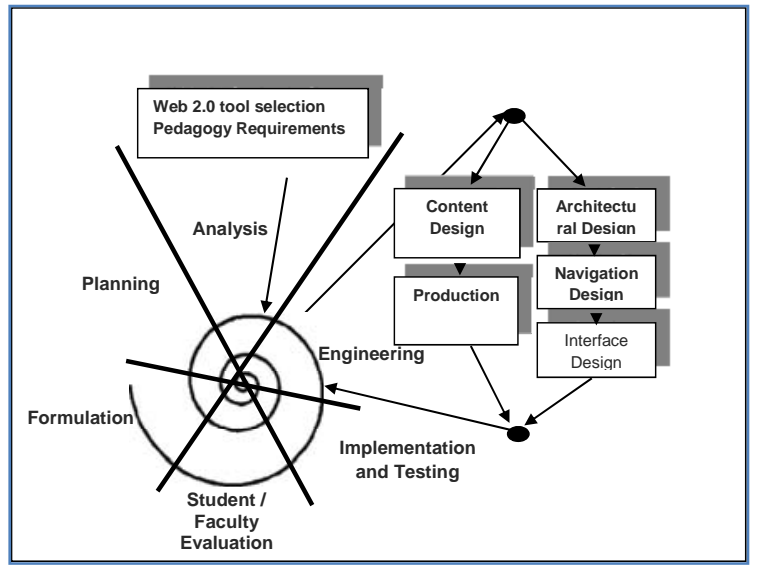

Figure 1. The enhanced development process

\section{Planning}

During the Planning phase, the total project cost and the risks associated with it are estimated. The project cost is calculated based on the formulated goals under the cost restrictions of the project and the additional Web 2.0 requirements. The risk estimation provides the project manager with a clear view of the potential weaknesses of the project. The Planning phase sets the timeframe for the implementation of the first increment as well as the process of the next increments.

\section{Analysis}

During the Analysis phase, the analyst following the classical approach studies the current system and processes and defines functional and non-functional requirements. Interviews with groups of students together with questionnaires formulated to include Web 2.0 functional requirements, are to be distributed among them are good examples of requirements elicitation methods. Focus questions with emphasis on the formation and use of social networks may also be used and examined by the eLearning engineers to capture valuable requirements (Table II). These are just a proposed set of key questions for the analyst to use as guidelines, but others may be used to enhance the set with application-specific questions, specific to the eLearning application under development. Finally, existing e-Learning templates may be used for quick identification of student and instructor preferences and gathering of requirements. Once all results are collected, are analyzed and evaluated they will be translated into functional and non-functional requirements of the system. 
TABLE II. WEB 2.0 TOOLS FOCUS QUESTIONS

\begin{tabular}{|l|}
\hline \multicolumn{1}{|c|}{ Focus Questions } \\
\hline Are students already familiar with Web 2.0? \\
\hline $\begin{array}{l}\text { Are users satisfied with using Web } 2.0 \text { tools for supporting their e- } \\
\text { learning activities? }\end{array}$ \\
\hline Are students ready to include Web 2.0 in the learning process? \\
\hline Will students adopt Web 2.0 tools? \\
\hline What are the students' feelings from using the new tools? \\
\hline How to develop appropriate pedagogy practices? \\
\hline How will the students be evaluated? \\
\hline How will the students evaluate the system? \\
\hline Are Web 2.0 used to complement to traditional e-Learning? \\
\hline Will students receive pedagogical support? \\
\hline Will the students feel isolated? \\
\hline Will the students perceived the experience as a rewarding one? \\
\hline
\end{tabular}

Requirements management follows, which deletes any duplication of the ones already found using the traditional method, or resolves conflicts resulting from contradictory requirements. Here the Web 2.0 functional and non-functional requirements are finalized. After updating the system requirements, their final form is used in the Engineering phase to support the e-Learning application development. The Web engineer designs the e-Learning application structure, the navigation mechanisms and the Web 2.0 tools, the interface and the content, based on the results obtained from the previous phase.

\section{Web 2.0 tool selection and evaluation}

In this phase the Web 2.0 tools will be selected. The analysis process is to be modified and adapted in order to include the selection and evaluation process of these tools and forms an integral part of the development process. Here the process subdivides in two paths: the tools and technologies available for selection to the trainer and the tools and technologies available to the trainee.

The trainer selects form the palette of tools as described in Table 1 and according to the indented audience, nature of the course and pedagogical objectives. The tools have been classified according to their function and popularity. If the trainer needs a highly interactive environment then tools such as forums and blogs should be used. If the trainer needs a more closed and structured environment the tools such as wikis and code repositories are more appropriate. If the trainer needs a more opinions from the trainees then blogs can be used.

The trainer also selects and defines Web 2.0 tools for the sole use of the learners although this can be achieved via traditional Web 2.0 applications. Additionally, the trainee is also able to select the Web 2.0 tools she/he will use and participate according to preference, ease of use, functionality, availability etc. The learner can now define an individual path to the learning process depending learning pace, scheduling requirements, access and availability of network resources and means of access.

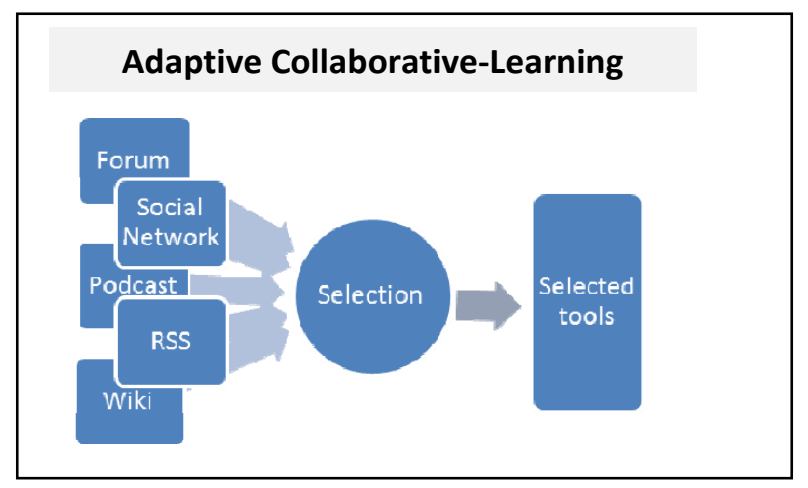

Figure 2. Adaptive Collaborative-Learning

\section{Engineering}

This phase includes the design and production of all material necessary for the e-Learning platform. Specifically, the following activities are included:

Architectural Design - Mapping of systems requirements with the Web 2.0 enhancements into the e-Learning system architecture representing the structure of data and program components. For the selected Web 2.0 tools the rules of use and access will be specified at this phase.

Navigation Design - Design of navigation pathways to enable users to access the e-Learning content and Web 2.0 tools by identifying navigation semantics of each user group and by defining the mechanics of achieving the navigation.

Interface Design - Development of a screen layout to serve as an effective communications medium prototype between the users (students and instructors) and the system. Web 2.0 tools interface design to facilitate the intended functionality.

Content Design - Design of the e-Learning application content, which includes text, graphics, images, video and audio data. The Web 2.0 tools as the enablers of social networks and the platforms for information exchange among the users will not require extensive content as it would be provided by the users.

Production - Development of the e-Learning application based on the information and designs gathered during the previous activities. All material is produced including the technical and pedagogical. Web 2.0 tools may be incorporated from existing ones or redeveloped if needed. 


\section{Implementation and Testing}

The implementation and testing phase includes the following three sub-activities:

Page Generation - Installation of the e-Learning application and its integration into the current system (if available), as well as the adaptation to the existing working procedures.

Testing - Testing of individual modules as well as of the e-Learning system as a whole employing black-box and testing-to-code techniques. As the eLearning system incorporates the Web 2.0 functionality its operation and effectiveness will be tested here.

Training - Demonstration and training of the system to the students and instructors, and creation of a user online tutorial and a reference guide. Although, tutorials are now forming an integral part of each tool, a comprehensive approach should be taken so that all parties will be able reap the benefits of the combined tools and technologies.

\section{Faculty/Student Evaluation}

In the evaluation phase we need to conduct ongoing evaluations of Web application development activities to ensure that each activity is performed in accordance to trainer learner requirements. The phase evaluates the interaction and usability of the eLearning context with the Web 2.0 tools by utilizing several methods, such as empirical testing, discount usability, cognitive and task analysis methods. Next, the phase evaluates each task and proposes new modifications and expansions that need to be incorporated to the next increment. Here all parties will be able to assess and evaluate the effects of the adopted tools and technologies. It is very important to keep our objectives clear and not to get carried away by using too many tools and thus overwhelming the learner. The medium is only the enabler of the pedagogical process and should be there to create an environment conductive to learning.

\section{Conclusions and Future Work}

In the paper we have presented an innovative framework for developing adaptive e-Learning systems based on Web 2.0 as a means to enhance the effectiveness of on-line learning systems in achieving their pedagogical goals. The proposed framework takes into consideration the pedagogy requirements and learning objectives. The framework through the Web 2.0 tool selection process is able to map the correct tools for the proposed pedagogical objectives and thus improve the effectiveness of the e-Learning systems.

\section{References}

[1] M. Khalifa, and R. Lam, "Web-based learning: Effects on learning process and outcome," IEEE Transactions on Education, 45(2), pp. 350--356, 2002.

[2] B. Mehlenbacher, C. R. Miller, D. Covington, J. S. Larsen, "Active and interactive learning on line: A comparison of web-based and conventional writing classes,” IEEE Transactions on Professional Communication, 43(2), pp. 166-184, 2000.

[3] M. Ebner, "E-Learning $2.0=$ e-Learning $1.0+$ Web 2.0?," In: The Second International Conference on Availability, Reliability and Security, pp. 1235--1239, 2007.

[4] Sigen Ma; Mingqin He; , "E-Learning Based on Web 2.0 Technical Characteristics," E-Business and EGovernment (ICEE), 2010 International Conference on , vol., no., pp.5431-5433, 7-9 May 2010.

[5] B. Leuf and W. Cunningham, "The Wiki Way. Quick Collaboration on the Web," Addison-Wesley, 2001.

[6] N. Towned, "Podcasting in Education", Viewfinder, Nr. 61, i-ii, 2005.

[7] Katerina Papanikolaou and Stephanos Mavromoustakos, "Web 2.0 Based e-Learning Development Process", Journal of the World Universities Forum, Volume 3, Issue 5, pp.117-126, 2009.

[8] S. He and P. Wang; , "Web 2.0 and Social Learning in a Digital Economy", IEEE International Symposium on Knowledge Acquisition and Modeling Workshop, pp. 1121-1124, 2008.

[9] Repnik, R.; Krasna, M.; , "WEB 2.0 in education: Do we really always need it?," MIPRO, 2010 Proceedings of the 33rd International Convention , vol., no., pp.843-848, 24-28 May 2010.

[10] Serrano, N.; Torres, J.M.; , "Web 2.0 for Practitioners," Software, IEEE , vol.27, no.3, pp.11-15, May-June 2010.

[11] Min Li; Zhengjie Liu; , "The Role of Online Social Networks in Students' E-Learning Experiences," Computational Intelligence and Software Engineering, 2009. CiSE 2009. International Conference on , vol., no., pp.1-4, 11-13 Dec. 2009.

[12] Lampe, C.; Resnick, P.; Forte, A.; Yardi, S.; Rotman, D.; Marshall, T.; Lutters, W.; , "Educational Priorities for Technology-Mediated Social Participation," Computer , vol.43, no.11, pp.60-67, Nov. 2010.

[13] Brown, S.; Flick, L.; Williamson, K.; , "Social Capital in Engineering Education," Frontiers in Education, 2005. FIE '05. Proceedings 35th Annual Conference , vol., no., pp.S3D, 19-22 Oct. 2005.

[14] Sterlocchi, S.; Ravarini, A.; "Change Management and Social Networks: The Enabling Role of e-Learning," Advanced Learning Technologies (ICALT), 2010 IEEE 
10th International Conference on, vol., no., pp.697-698, 57 July 2010.

[15] Angelaccio, M.; Buttarazzi, B.; "A Social Network Based-Enhanced Learning System," Enabling Technologies: Infrastructures for Collaborative Enterprises (WETICE), 2010 19th IEEE International Workshop on, vol., no., pp.94-95, 28-30 June 2010.

[16] Yonggu Wang; Xiaojuan Li; "Social Network Analysis of Interaction in Online Learning Communities," Advanced Learning Technologies, 2007. ICALT 2007. Seventh IEEE International Conference on, vol., no., pp.699-700, 18-20 July 2007.

[17] Tak-Wai Chan; , "Social design of network learning society," Computers in Education, 2002. Proceedings. International Conference on, vol., no., pp. 1- 5 vol.1, 3-6 Dec. 2002.

[18] Singh, G.; O'Donoghue, J.; "A study of sociallearning networks of students studying an on-line programme," Advanced Learning Technologies, 2001. Proceedings. IEEE International Conference on, vol., no., pp.263-266, 2001.

[19] Sommerville, I. and Sawyer, P.: "Requirements Engineering: A good practice guide”, J. Wiley and Sons, 1997.

[20] Pressman, R. S. "Software engineering: A practitioner’s approach”, McGraw-Hill, London, 2000. 